\title{
Implicaciones Socio-ambientales del Conflicto en Colombia
}

\section{Social and Environmental Implications of the Conflict in Colombia}

\author{
Martha Inés Moreno Medel \\ Politécnico Grancolombiano, Calle 57 N. 3 - 00 Este, Bogotá, Colombia \\ mimorenom@poligran.edu.co
}

DOI: https://doi.org/10.33017/RevECIPeru2016.0016/

\begin{abstract}
Resumen
El conflicto genera devastación, desplazamiento, desempleo y crisis alimentaria; en las mesas de negociación se están buscando soluciones, pero también propician conflicto, las personas desplazadas y trabajadoras de escasos ingresos no van a tener los mismos beneficios, esto genera inequidad; uno de los factores que contribuye a superar este impacto es la educación desde el cerebro, mirándolo integralmente. La educación se debe fundamentar en la construcción del cerebro tríadico tetra-nivelado (tres predominancias en cuatro niveles de desarrollo); utilizando programas de capacitación basadas en el desarrollo de competencias del cerebro tríadico; actualmente cada predominancia cerebral busca su propio beneficio y es necesario deshacer conocimientos para poder tener un cambio de mentalidad, donde cada actor del conflicto logre empatizar con quienes fueron sus adversarios tras un bien común, la sobrevivencia en condiciones dignas y la conservación del planeta; los estudios realizados hasta el momento y las estrategias implementadas han sido desde el currículo tradicional. En el conflicto el factor principal es el ser humano; es inviable una paz duradera si no se enfrenta a un proceso de desaprender y adquirir aprendizajes nuevos desde el cerebro integral: la predominancia izquierda lógica que es desde donde se ha venido adelantando el proceso de negociación, derecha para desarrollar la parte afectiva, creativa del entorno y la predominancia central reptiliano donde está la sobrevivencia.
\end{abstract}

Descriptores: Cerebro tríadico tetranivelado, conflicto, postconflicto, inequidad, empatizar, agroecología.

\begin{abstract}
The conflict has generated devastation, forced displacement, unemployment and even food crisis; Solutions are being sought through the peace talks, but these at the same time create other type of conflict because displaced people and workers with limited resources are not going to have the same benefits, which generates inequity. Education should be based on the construction of the triadic brain tetra-level (three predominances in four levels of development); using training programs that use as a base the skills development of the triadic brain; Nowadays each brain predominance looks for their own benefit and it is needed to dissolve some knowledge to be able to have a mentality change, where everyone that has been a victim in conflict is able to empathize with their opponents in search of general welfare, survival in decent conditions and the planet conservation, studies and the implemented strategies so far have been from the point of view of the traditional curriculum. In conflict the main factor is the human being; it is unsustainable for peace to last if we don't face the process to dissolve some knowledge and learn something new from the integral brain: the left logical predominance where all the peace talks have come from, the right one to develop the affective, creative part of the environment and the central reptilian predominance where survival is located.

Agroecology forms an innovative tridimensional trend to implement (Science, Practice, Social Movement and/or Political), where the changes of scientific and technologic paradigms act and work in constant reciprocity to achieve a social impact.
\end{abstract}

Keywords: Triadic Brain tetra-level, Conflict, Post-conflict, Inequity, Empathize, Agroecology 


\section{Introducción}

El 18 de noviembre de 2012, se instaló en Oslo Noruega la mesa de dialogo entre la FARC y el Gobierno Colombiano, mesa que se aplazó hasta el 19 de noviembre del mismo año, en la Habana, Cuba; desde esta época se han venido discutiendo diversos temas entre estos el Impacto de la guerra en el medio ambiente y las posibles soluciones para minimizar este impacto, es por esta razón que las autoridades Internacionales y Nacionales se han preocupado por minimizarlo, a continuación se plasman algunas de los impactos, los planes de acción implementados y una propuesta para preparar a los involucrados para el post conflicto.

El conflicto en Colombia inicia desde la década de los sesenta, durante este tiempo entre los factores que se han afectado considerablemente han sido los recursos naturales con atentado a los oleoductos, que generan catástrofes ambientales.

Desde el punto de vista social se ha generado desplazamiento a las grandes ciudades incrementando el desempleo, el hambre y la vulnerabilidad de los derechos fundamentales.

Las implicaciones socio-ambientales y las estrategias para minimizar este impacto a través de la educación y divulgación, utilizando programas de capacitación que tengan como base el desarrollo de competencias del cerebro Tríadico, con el fin que los actores del conflicto puedan prepararse para el postconflicto.

Con este fin, desde los planes gubernamentales y el punto de vista del Desarrollo Sustentable es necesario identificar el impacto del conflicto en las zonas del país que han vivido de primera mano sus consecuencias.

El valor agregado que se da en el presente artículo es que los estudios realizados hasta el momento han sido desde el punto de vista del currículo tradicional, la propuesta que se realiza es que la nueva educación, se debe fundamentar en la construcción del Cerebro tríadico Tetra-nivelado; donde cada predominancia cerebral busca su propio beneficio y es necesario deshacer conocimientos para poder tener un cambio de mentalidad donde cada actor del conflicto logre empatizar con quienes fueron sus adversarios en el conflicto tras un bien común que es la conservación del planeta.

En la mayoría de los países que han atravesado por conflicto armado [18], uno de los atentados que genera más víctimas silenciosas son los atentados contra los recursos naturales; es así como en Nicaragua el atentado al cerro Mokorón de 270 metros de altura cubierto por 17 hectáreas de bosque tropical seco, originó la Ley 559 "Ley Especial de Delitos contra el Medio Ambiente y los Recursos Naturales" [28].

En Honduras a través de la Ley General del Ambiente promulgada en el Decreto 104-de 1993 se imponen sanciones por los atentados al medio ambiente, no obstante ministro de Agricultura y Ganadería, Jacobo Paz, fue acusado de los delitos de "escombro, daños a la fauna y roturación en perjuicio del Estado de Honduras", cuando se desempeñaba como gerente de la Granja Marina San Bernardo, en 2013.

En Argentina, el mayor desastre ecológico no ha sido causado por el terrorismo sino por la minería; El 13 de septiembre de 2015 Barrick Gold admitió que derramó más de un millón de litros de solución con cianuro en el Rio Potrerillos; por estos hechos fue investigada y sancionada a través de la aplicación de la Ley 25.675 denominada "Política Ambiental Nacional".

\section{Antecedentes}

El presente estado del arte busca suministrar información sobre las tendencias actuales en investigaciones sobre el impacto socio-ambiental del conflicto en Colombia para esto es necesario hablar de conflicto socio-ambiental y revisar los diversos factores que lo impactan.

Del Viso [24], distingue los conceptos de conflicto ambiental como una degradación en la explotación de los recursos naturales renovables, una presión sobre la capacidad de sumidero del ecosistema y un empobrecimiento del espacio de vida y conflicto socio-ambiental.

Según Fontaine, [10] los conflictos en tema ambiental son causados por la actividad petrolera, minera y forestal y son realizadas por parte de movimientos ambientalistas; de otra parte Páramo [13], argumenta que se presentan conflictos socioambientales, entre habitantes de un mismo territorio, las autoridades locales y ambientales, alrededor de problemas como la tala de bosque, la disposición de basuras o la contaminación específica de un lugar; mientras que en el análisis del conflicto en Colombia realizado por Kurtenbach [30], se menciona que el acceso desigual a la tierra y a otros recursos 
naturales es la causa fundamental del conflicto socio-ambiental en Colombia.

Novoa [17], analizando la discordia entre la Occidental de Colombia (OXY) y la comunidad indígena U'wa, puso en el centro de la discusión aspectos como el respeto a la integridad cultural, el carácter multiétnico y pluricultural de Colombia y Vinyamata [8], identificó como causas del conflicto socio-ambiental, la insatisfacción de las necesidades básicas de sectores de la población mundial.

En Colombia el conflicto con las FARC [9] ha generado atentados contra los oleoductos y esto ha ocasionado desplazamiento, mortandad de fauna y flora; otro factor que ha incidido es la explotación de oro a cielo abierto y uso desmedido de plomo y la producción ilícita de sustancias alucinógenas [2].

En el año 2015 Torres y Ruiz [15], analizan el extractivismo neoliberal en Colombia y Perú desde 1990, mostrando que la gestión de los recursos mineros se deriva de un modelo que favorece la acumulación privada y extranjera de capital; acompañado de políticas de militarización de los territorios mineros y de supresión de la movilización social y concluyeron que las políticas para incentivar la inversión extranjera directa en minería han generado graves impactos ambientales, violación de derechos humanos y profundización de conflictos socio-ambientales, afectando de forma espacial a comunidades étnicas. [15].

Pérez [21] levanta un inventario y mapeo inicial de los principales conflictos socio-ambientales en Colombia, habiéndose documentado inicialmente 72 casos distribuidos por todo el territorio nacional, se observó que los campesinos, la población urbana y los indígenas son los grupos poblacionales más perjudicados, los sectores mayores generadores de impacto son en términos de área afectada son la energía fósil y la infraestructura; en términos de población afectada son la biomasa, la minería y los residuos sólidos y en términos de recursos invertidos la generación de energía y la minería [25,27,33] .

Lavaux [31] atribuye a los conflictos armados, el terrorismo ambiental que conlleva a contaminación de fuentes hídricas, aridez en los suelos hasta llegar a la muerte del ecosistema.

Pérez [20] Menciona en su tesis que el comercio internacional traslada los costos sociales y ambientales a territorios y zonas específicas y grupos poblacionales particulares que se afectan social y ambientalmente y genera conflictos ecológicos.
Quimbayo [14], Analiza el caso de la ciudad de Bogotá Distrito Capital, Colombia, y su área metropolitana, donde existen espacios naturales como cerros, humedales y ríos en donde aún persisten importantes muestras de biodiversidad y que, como ecosistemas, permiten la sostenibilidad del territorio, pero los intereses particulares han originado asentamientos que han atentado contra estos recursos, esta afirmación se puede reafirmar con lo manifestado por Andrade [12] y Van der Hammen [34], en cuanto a que las condiciones biofísicas e hidrogeológicas de alta montaña existentes en teoría representarían limitantes para la urbanización y para la minería en los cerros de Bogotá [6]. No obstante, la ciudad se ha erigido como el mayor centro urbano, político y económico de Colombia y la respuesta institucional ha sido históricamente errática 0 nula en implementar medidas de conservación e inclusión social [22].

El paramilitarismo también originó desalojo presionó por vía de las armas la venta de predios, y controló la movilización y la denuncia de los líderes a través de su asesinato, amenaza y desplazamiento [7].

Partiendo que el conflicto se basa en el factor principal que es el ser humano es necesario afrontar los factores inherentes a él, es por esta razón que se debe abordar desde lo inherente a este; no es viable que el postconflicto sea duradero si no se enfrenta a un proceso de desaprender y volver a los procesos de aprendizaje y esto sólo se logra desde el cerebro de manera integral, la predominancia izquierda lógica que es desde donde se ha venido adelantando el proceso de negociación, derecha para desarrollar la parte afectiva, el sentido artístico y la parte subliminal del entorno y la predominancia central rectiliano donde está el instinto se sobrevivencia; por tal razón se van a abordar estudios adelantados sobre este tema.

MacLean [26], con su teoría evolutiva del cerebro triple propone que el cerebro humano fue en realidad tres cerebros en uno: el reptiliano, el sistema límbico y la neocorteza; enmarcando en tres etapas de evolución el desarrollo del cerebro; el cerebro reptil concebido como el tallo cerebral, que controla los elementos básicos de superviviencia, el cerebro paleomamífero que comprende el sistema límbico que permite que los procesos de sobrevivencia interactúen con su entorno y el cerebro neomamífero o neocorteza que regula las emociones específicas.

Sperry [29], en su trabajo sobre el "Cerebro Dividido" analizando dos estructuras cerebrales y en su teoría del dominio del lado izquierdo o derecho cerebro, 
dice que cada lado controla los distintos tipos de pensamiento. Además, la gente prefiere un tipo de pensamiento sobre el otro. Por ejemplo, una persona que utiliza más la parte izquierda del cerebro se dice que es más lógico, analítico y objetivo, mientras que una persona que es utiliza más el lado derecho, se dice que es más intuitivo, reflexivo y subjetivo.

Como profundizador de los estudios del cerebro Triuno, Gregori [36], sociólogo e investigador brasileño analiza la relación entre cerebro y educación para plantear un cambio fundamental en la sociedad a través del modelo tríadico tetra nivelado.

Según De Gregori [37], el ser humano vive en una lucha de sus tres cerebros y la sociedad forma parte de esta lucha; por esta razón es necesario que el primer factor al que se debe dar solución es a las necesidades básicas que son las que dependen del cerebro central; el presente proyecto pospone una propuesta para dar solución a esto; cubiertas estas necesidades se puede iniciar la etapa de aprendizaje por el cerebro izquierdo; esta etapa puede estar cubierta en parte por las políticas gubernamentales y de allí se logra también el aprendizaje por el cerebro derecho, que se enmarcada en el sentido místico y en este caso de la relación naturaleza y ser humano, y del cerebro central con su sentido de sobrevivencia para que valore la importancia del medio ambiente como medio para lograrla.

\section{La Educación Agroecológica como solución en el post-conflicto}

Una vez analizado el contexto en América Latina y específicamente en Colombia se puede determinar que la principal solución para el postconflicto es la educación integral del ser humano para esto se retomarán las principales acciones que ha tomado el gobierno colombiano.

\subsection{La Educación ambiental en Colombia}

En Colombia en la Constitución Política de Colombia de 1991, dice: "es obligación del Estado y de las personas proteger las riquezas culturales y naturales de la Nación", y "es deber del Estado proteger la diversidad e integridad del ambiente, conservar las áreas de especial importancia ecológica y fomentar la educación para el logro de estos fines".

Con el fin de cumplir los preceptos de la carta magna se han venido implementando estrategias y es así como entre 1992 y 1994 se realizó la etapa de exploración, luego entre 1994 y 1995 se llevó a cabo la etapa de profundización donde se realizó reflexión teórico - Conceptual sólo con el manejo de la información, el gran avance se dio en la ley 115 de 1994 (Ley General de Educación); la cual en el artículo 5, inciso 10 , define como uno de los fines primordiales de la educación "La adquisición de una conciencia para la conservación, protección y mejoramiento del medio ambiente, de la calidad de vida, del uso racional de los recursos naturales, de la prevención de desastres, dentro de una cultura ecológica...". En 1995 inicia la etapa de proyección se dio inicio mediante formulación, difusión e implementación de los primeros lineamientos formales en Educación Ambiental, tomando como base el papel que juegan los padres, maestros, alumnos [23].

El 25 de enero de 2015 el ministro de Ambiente Gabriel Vallejo López explicó "La educación ambiental es uno de los cuatro pilares que definió el Ministerio de Ambiente y Desarrollo Sostenible para conformar la política ambiental de Colombia en 2015 y es que una de las grandes apuestas es seguir educando a los colombianos para que se interesen y tomen conciencia sobre la importancia que tiene hacer uso de los recursos naturales de forma racional".

En América Latina, una de las dificultades que encuentran las personas víctimas de los conflictos socio-ambientales es el desplazamiento, por lo general de la población campesina, dejando atrás tierras productivas y se ven en la necesidad de trasladarse a las grandes ciudades donde se ven enfrentados al desempleo, la privación de elementos de primera necesidad y la pobreza, esto genera escasez alimentaría para ellos y para la población en general porque ya no hay quien cultive la tierra y Bogotá no dista de estas dificultades por ser Ciudad Capital.

La agroecología surge como una solución, permitiendo logros importantes como la soberanía alimentaria basada en la conservación de los recursos naturales y el empoderamiento local, regional y nacional de organizaciones y movimientos campesinos [19].

En estas circunstancias, la educación juega un papel importante, porque a través de ella se ha concebido la agroecología como una solución a los problemas alimentarios y económicos; se ha fomentado el cultivo en terrazas y antejardines de los hogares y en los establecimientos educativos, que en primera instancia cubre las necesidades de las familias y en algunos casos permite un ingreso adicional a través de la venta 
de productos de excelente calidad, a bajos precios y benéficos para la salud.

Las instituciones educativas y gubernamentales han emprendido procesos de capacitación y acompañamiento generando de esta forma una opción para cubrir las primeras necesidades.

\subsection{Sustentabilidad agroecológica}

Partiendo del carácter tridimensional de la agroecología, como ciencia, como práctica y como movimiento social y/o político [35], se concluye que la agroecología conforma un novedoso fenómeno, donde los cambios de paradigmas científicos y tecnológicos actúan y se construyen en constante reciprocidad.

En Latinoamérica, la agroecología académica ha tenido una expansión que se expresa en el número de publicaciones, instituciones, congresos y nuevas sociedades científicas regionales y nacionales $[3,19]$.

Un elemento clave en la dimensión académica ha sido la formación de cientos de nuevos agroecólogos dentro de lo que podría llamarse la corriente social de la agroecología de España [ y han generado inquietud y compromiso para implementar estas soluciones en las ciudades como Bogotá.

El desarrollo de conocimiento agroecológico, ha surgido en varios países y actualmente se puede hablar de los huertos urbanos orgánicos [19] y el Movimiento de Campesino a Campesino impulsado por la Asociación Nacional de Agricultores Pequeños (ANAP) [4] y variedad de eventos internacionales y de estos eventos nació la ANA (Articulacao Nacional de Agroecologia), que opera como un instrumento estratégico entre las organizaciones sociales y políticas de productores, la esfera técnica y de investigación aplicada y las instituciones académicas [35].

El auge más importante en América Latina y el Caribe es el involucrar a los niños y adolescentes, no solo de las zonas rurales sino también a los de las ciudades en la importancia de aprovechar espacios disponibles de las ciudades donde antes no se veía viable, para la generación de productos agrícolas de primera necesidad; en Bogotá, los proyectos de agricultura urbana son impulsados principalmente por la universidad de los Andes, la del Rosario, la Corporación Universitaria Minuto de Dios, Corpoíca, el Jardín Botánico de Bogotá y el Sena; no obstante algunos centros educativos han generado este tipo de iniciativas y son los casos que nos ocupan en el presente artículo.
En Colombia los Colegios han mostrado algunos casos de éxito, que han permitido a población vulnerable y desempleada, generar alimentos para sus hogares y conseguir recursos para cubrir sus necesidades básicas.

Las Instituciones educativas se han preocupado por fomentar el conocimiento de la agricultura urbana como fuente de recursos para los hogares, este tipo de agricultura no requiere una alta inversión, ni tecnología, por el contrario con unos pocos recursos o con algo de financiación los hogares e instituciones educativas pueden contar con lo necesario para cultivar productos de primera necesidad; adicionalmente pueden generar ingresos, pero es necesario que esto se difunda hasta los hogares y desde ellos; las comunidades que han visto las ventajas de estos proyectos pueden corroborar que su calidad de vida ha cambiado considerablemente.

Otro factor importante es que se ha disminuido el mal uso de los residuos orgánicos que generaban contaminación y se han aprovechado en proyectos como la lombricultura que genera abono para las huertas; de tal forma que los productos agrícolas son sanos, porque carecen de químicos que disminuye su calidad nutricional.

Cabe la pena destacar que estos proyectos han contribuido a mejorar el desarrollo alimentario de las comunidades que los han implementado, debido a que antes no tenían acceso a algunos productos agrícolas por ser costosos y que por su situación de desplazamiento y desempleo no podrían adquirir; por el contrario se han constituido en proveedores de los mismos para sus vecinos ofreciéndolos a menor costo que el ofrecido en las plazas de mercado.

\subsection{La lombricultura solución a problemas ambientales y para la crisis alimentaria}

Uno de los problemas en la crisis ambiental es el uso inadecuado de los desechos biodegradables cuando no son seleccionados adecuadamente en la fuente, no obstante cuando hay una adecuada selección estos desechos son utilizados para la generación de biomasa para la alimentación de animales y humus para fertilización de suelos a través de la lombricultura de tal forma que disminuye la utilización de fertilizantes de origen químico, generando productos agrícolas más saludables.

Con la lombricultura se genera proteína para alimentación de peces y gallinas y en algunos casos 
aunque no se ha masificado también se ha utilizado para alimentación humana [32], la carne de lombriz tiene mayor nivel de proteína que la carne de res y los frijoles; adicionalmente disminuye los desperdicios orgánicos toda vez que estos son utilizados para el proceso de sostenimiento de estas lombrices y como residuo se genera el humus para la fertilización de suelos; estos cultivos son muy económicos porque no requieren preparaciones espaciales son al aire libre con lechos colocados en cualquier tipo de terreno.

Según estudios realizados por la bióloga Ana García [1], del Departamento de zoología y antropología Física de la Universidad Complutense de Madrid, la lombriz de tierra es ingrediente fundamental de la dieta de las embarazadas de comunidades de indios amazónicos y contiene gran aporte en calcio "Tan solo treinta gramos de esta enorme lombriz suponen el aporte diario de calcio necesario para las embarazadas y para el buen desarrollo del feto" dice la bióloga".

Otro uso para la alimentación de poblaciones vulnerables es como producto alimenticio para niños a través del procesamiento en forma de galletas [5], estas suplen el valor nutricional de la leche y de la carne a unos costos inferiores a estos productos.

Se han realizado varios estudios y proyectos con el fin de masificar el consumo de la lombriz, entre ellos se puede mencionar el realizado por la universidad Nacional donde se elaboraron salchichas a partir de la tilapia y la lombriz; $40 \stackrel{\circ}{\circ}$. [16] "Nuestros climas templados son ideales para su propagación".

\section{Conclusiones}

Los conflictos ambientales en Colombia, han generado devastación, desplazamiento, desempleo y crisis alimentaria de la población que se ha sido despojada de su territorio y se ha visto en la necesidad de migrar a las ciudades principales; algunas de estas personas.

A través de las mesas de negociación se están buscando soluciones; pero entre las planteadas están dar vivienda y subsidios a los desmovilizados pero esto está generando otro tipo de conflicto toda vez que las personas que han sido desplazadas no van a tener los mismos beneficios y otras personas cabeza de familia con varios hijos y empleadas que devengan un salario mínimo nunca van a lograr los mismos beneficios; la sugerencia del presente artículo es que estos subsidios sean únicamente mientras se logran ubicar y luego se les de las mismas posibilidades de los desplazados y se fomente la agroecología urbana y la lombricultura para procesos de fertilización de suelos; es necesario que inicialmente a esta población se les incentive en afirmar este cultivo para procesos alimentarios de peces y aves de corral y para el consumo humano; estrategia que puede ser implementada no sólo en Colombia sino en cualquier región del mundo que atraviese este tipo de crisis.

Lo antes mencionado debería ser una política gubernamental debido a que el país se está preparando para el posconflicto y no es viable la paz con hambre; adicionalmente no hay fuentes de trabajo.

Si se le da la opción a las personas desempleadas más que un subsidio, la posibilidad de generar sus propios recursos a través de la agroecología y fertilizando los suelos con la lombricultura y se masifica como alimento para la cría de especies alimenticias como peces y aves de corral y se fomenta el consumo de productos a base de lombriz, se está dando una solución de fondo a la crisis alimentaria del país y de otras regiones del mundo.

De otra parte, además de ser estos recursos utilizados para cubrir las necesidades básicas de las familias que se involucren en estos proyectos, pueden ser fuentes de ingresos al ser comercializados, debido a su alta calidad por ser producidos sin insumos químicos y sin afectar el medio ambiente.

Si a la educación tradicional se le involucra el factor de Desarrollo Sustentable y el concientizar a la persona de actuar como ciudadana y se deja a un lado el instintito paternalista del estado y la iglesia de cubrir las necesidades básicas y la involucra en el proceso para ser parte activa de este, acogiendo la propuesta que ser participe en el cambio radical volviendo a sus regiones y se incentive la agricultura; el $90 \%$ de los desmovilizados han sido arrancados del campo y lo óptimo es que vuelvan a él pero cubriendo sus necesidades con subsidios que fomenten la reconstrucción de la tierra; no que lleguen a las grandes ciudades a ser un problema más de sobre población y desempleo.

\section{Agradecimientos}

Agradezco en primera instancia a Dios, por la vida la salud y permitirme laborar en una institución donde puedo desarrollarme como persona y profesionalmente, a la Decana de la Facultad de Ciencias Administrativas, Económicas y Contables, al Director del Programa de Contaduría Pública, a la Directora Académica de Educación Virtual, a los 
Semilleristas, "La virtualidad en la pedagogía desde las competencias tríadicas para el pos conflicto" del Politécnico Grancolombiano, por el apoyo y acompañamiento en la presente investigación.

A mis hijos Angélica M., Gustavo A. y Diana C., por su paciencia, por estar presentes siempre y por ser el motor de todos los proyectos que emprendo.

\section{Referencias}

[1] A. García, Biodiversidad y conservación en artrópodos. Madrid, España. Recuperado de: http://www.foro.salvatuvida.com/viewtopic.php $? \mathrm{t}=4881$. (2013).

[2] A. Quiñones. Geopolítica de los conflictos socio ambientales: resistencia a la expansión minera. Memoria y Sociedad 19, n.․ 39. Recuperado de: http:// dx.doi.org/10.11144/Javeriana.mys1939.gcsr. (2015). p. 73-92

[3] B.G. Ferguson, H. Morales. Latin American agroecologists build a powerful scientific and social movement. Journal of Sustainable Agriculture 34: 2010. p. 339-341.

[4] B. Machin, a. Roque, D. Avila, P. Rosset. Revolución Agroecológica: el Mov Revolución Agroecológica: el Movimiento de Campesino a Campesino de la ANAP en Cuba. www.abyayalacolectivo.com/web/comparti r/noticia/revolucion-agroecologica. (2010).

[5] B. Mendez. ¿Quien dijo que comer lombrices era malo? ¡Nuevas lombrigalletas! mas que pura harina. Instituto Politécnico Nacional. Recuperado

de: http://noticias.universia.net.mx/ciencia-nntt/noticia/2005/01/19/111953/dijo-comerlombrices-era-malo-nuevaslombrigalletas-mas-pura-harina.html. (s.f.).

[6] C. Ordoñez, A.E. Angel, y D.P. Lozano.. A través de la ventana: una apreciación paisajística de los efectos de la minería en los cerros de Bogotá, En: Toro Pérez, C., Fierro Morales, J., Coronado Delgado, S. y Roa Avendaño, T. (Eds.) Minería, Territorio y Conflicto en Colombia, Universidad Nacional de Colombia, (2013). p. 381-398.

[7] D. Muñoz, A. Idárraga, y H. Vélez, Conflictos socio-ambientales por la extracción minera en Colombia: casos de la inversión británica. Bogotá, Colombia: CENSAT Agua Viva. (2010).

[8] E. Vinyamata. Manual de Prevención y Resolución de Conflictos. Conciliación, Mediación, Negociación. Editorial Ariel S.A., Barcelona, (1999). P. 67-68.
[9] G. Ayala. Ambiente y Conflicto Armado Interno, una Apuesta Interdisciplinar. (2014).

[10] G. Fontaine. Enfoques Conceptuales y metodológicos para una sociología de los conflictos ambientales. En: Cárdenas, Martha y Rodríguez, Manuel. Guerra, Sociedad y Medio Ambiente. Foro Nacional Ambiental. Bogotá, (2004). P. 506.

[11] G, Guzmán, M, González, E, Sevilla. Introducción a la Agroecología como Desarrollo Rural Sostenible. Ediciones Mundi Prensa. (2000).

[12] G.I. Andrade, F. Remolina, y D. Wiesner, Assembling the pieces: a framework for the integration of multi-functional ecological main structure in the emerging urban region of Bogotá, Colombia, Urban Ecosystems, Springer, Volume 16, Issue 4, (2013), p. 723739.

[13] G. Páramo. Ecosistemas Naturales Colombianos en Conflicto. En: González, Juan Manuel y otros. La Manzana de la Discordia. Debate sobre la naturaleza en Disputa. T.M. Editores. Eco fondo. Segunda edición. Santafé de Bogotá, (1998).

[14] G. Quimbayo. Movimientos sociales, políticas y conflictos ambientales en la construcción de ciudad: El caso de Bogotá. Ecología Política Cuaderno de debate Internacional, (2014). P. 104-107.

[15] I. Torres y G. Ruiz. Extactivismo Neoliberal Minero y Conflictos Socio-ambientales en Perú y Colombia. Ambiente y Sostenibilidad. Revista del Doctorado Interinstitucional en Ciencias Ambientales, (2015). P. 3-15.

[16] J. I. Hleap,. Tilapia y Lombriz, juntas en una nutritiva salchicha. El tiempo. Recuperadode: http://www.unperiodico.unal.edu.co/dper/articl e/tilapia-y-lombriz-juntas-en-una-nutritivasalchicha.html. (2012)

[17] J. Novoa, U'wa/Oxy: el conflicto continúa. En: González, Juan Manuel y Otros. La Manzana de la Discordia, debate sobre la naturaleza en disputa. Segunda edición. ECOFONDO, Tercer mundo editores S.A. Santafé de Bogotá, Colombia. (1998). P. 221223.

[18] J. Rojas. Conflictos ambientales por medidas de mitigación al cambio climático en territorio Wayuu: el Parque Eólico Jepirachi, 19992011, Colombia. Bogotá: Instituto de Estudios Ambientales, Universidad Nacional de Colombia. (2012). 
[19] M. A. Altieri, La revolución agroecológica de América Latina: Rescatar la naturaleza, asegurar la soberanía alimentaria y empoderar al campesino. (2010). El Otro Derecho no. 42.

[20] M. Pérez. Conflictos ambientales en Colombia: inventario, caracterización y análisis. En Garay Salamanca, L J (Dir.). Minería en Colombia. Control público, memoria y justicia socio-ecológica, movimientos sociales y posconflicto. Contraloría General de la República. (2014).

[21] M. Pérez, Conflictos ecológicos distributivos Asociados a la actividad exportadora Colombiana. Tesis Doctoral. Comercio Internacional y Medio Ambiente en Colombia. Barcelona: Universidad Autónoma de Barcerlona, (2006). p.191-220.

[22] M. Ruiz. Lineamientos para una historia agroambiental de la Sabana de Bogotá (18501999), En: G. Palacio (Ed.), Historia ambiental de Bogotá y la Sabana, 1850-2005, Bogotá, Instituto Amazónico de Investigaciones-Universidad Nacional de Colombia, (2008). p. 48-71.

[23] M. T. Carrasco. La Educación Ambiental En Colombia: "Un Contexto de Transformación Social y un proceso de Participación en Construcción, a la Luz del Fortalecimiento de la Reflexión- Acción". Bogotá: Programa de Educación Ambiental Ministerio de Educación. (2010).

[24] N. Del Viso, Aspectos culturales de los mecanismos comunitarios de resolución de conflictos socioecológicos y percepciones sobre el uso de la tierra. Chile: IECTA: IQUIQUE. (2010).

[25] OEA. Reforma Política sobre energía renovable en América Latina y el Caribe. Serie sobre elementos Políticos. Número 5. Recuperado de: https://www.oas.org/dsd/policy_series/5_s pa.pdf. (2004)

[26] P. MacLean. The triune brain in evolution: role in paleocerebral functions. Nueva York: Plenum Press. ISBN 0-306-431688. OCLC 20295730. (1990)

[27] República de Colombia. Ley 1715. Por medio de la cual se regula la integración de las energías renovables no convencionales al Sistema Energético Nacional. (2014).

[28] República de Nicaragua. Ley 559. Ley Especial de Delitos contra el Medio Ambiente y los Recursos Naturales. (2005)
[29] R. W. Sperry. Regulative factors in the orderly growth of neural circuits. Growth (Suppl 10): (1951). P. 63-87

[30] S. Kurtenbach. Análisis del Conflicto en Colombia. FES. Bogotá, Colombia, (2005). Pág. 16.

[31] S. Lavaux. Degradación ambiental y conflictos armados: las conexiones. Grupo de Investigación sobre Seguridad. - Bogotá: Centro Editorial Universidad del Rosario, (Facultades de Ciencia Política y Relaciones Internacionales; Documento de Investigación; 7) Incluye bibliografía. ISSN: 1692. (2004.) p. 30.

[32] S. Z. Echeverry, Proteinaas alimentarias alternativas: Eficiencia Nutricional de la Harina de Lombriz Roja Californiana. (V. Relaciones Internacionales, Ed.) Pasto, Nariño, Colombia. Recuperado de: http://blade1.uniquindio.edu.co/uniquindio////// eventos/colombo-

hispanos 1/doc/conclusiones-mesas-

tematicas/Proteinas

ROTEINAS ALIMENTARIAS ALTERNATIVA S EFICIENCIA NUTRICIONAL DE LA HA RINA_DELOMBRIZ_ROJA_CALIFORNIANA. pdf. (s.f.).

[33] T. González, J.A. Marin. CONVENIO ATN/FM-12825-CO Componente I Proyecto inversiones catalizadoras para energía geotérmica Promoción de criterios de mercado para las energías renovables no convencionales a través de la eliminación de barreras para su desarrollo. Ministerio de Minas y Energía Unidad de Planeación Minero Energética - UPME. REPÚBLICA DE COLOMBIA, (2015).

[34] T. Van der Hammen. Historia, Ecología y Conservación de Ecosistemas Altoandinos y de Páramo hacia la realización de una estructura ecológica para la región (Cuenca Alta del Río Bogotá), En: Memorias del Encuentro Internacional de Ecología Regional Aplicada a la Conservación de la Flora y los Ecosistemas Alto Andinos y de Páramo, Bogotá, Colombia. Academia Colombiana de Ciencias Exactas, Alcaldía Mayor de Bogotá, Departamento Técnico y Administrativo del Medio Ambiente, Jardín Botánico de Bogotá José Celestino Mutis, (2006). p. 39-50.

[35] V. Toledo. La Agroecología en Latinoamérica: Tres revoluciones, una misma transformación. U. N. Centro de Investigaciones en Ecosistemas, Ed. Agroecología 6:37, (2012). P. 37-46. 
[36] W. De Gregori, Neuroeducación para el éxito, Construcción - Productividad - Decadencia de los tres cerebros y sus competencias. Recuperado:

http://books.google.com.br/books/about?id=0 WmsBQAAQBAJ\&redir_esc=y DE GREGORI, [2003]: Construcción familiar - escolar étnica de los tres cerebros. Bogotá: Kimpress. (2014).

[37] W. De Gregori, E, Volvato, Capital Intelectual:
Administración Sistémica, Manual de Juegos de Cooperación y Competencia. Bogotá: Mc Graw Hill, (2001). 